\title{
Propagation of interacting force chains in the continuum limit
}

\author{
Yael Roichman and Dov Levine \\ Department of Physics, Technion, Haifa 32000, Israel \\ Irad Yavneh \\ Department of Computer Science, Technion, Haifa 32000, Israel
}

(Dated: September 13, 2018)

\begin{abstract}
We study the effect of mergers in the force chain model describing the stress profile in static granular materials. Combining numerical and analytical calculations we show that granular materials do not generally behave in an elastic-like manner, however they may under specific conditions, which are elaborated. Non-elastic behavior resulting from the non-linearity of the full force chain model is discussed.
\end{abstract}

PACS numbers: $45.70 . \mathrm{Cc} ; 83.80 . \mathrm{Fg}$

A striking characteristic of stress transmission in granular matter is the network of highly singular lines, termed force chains, along which stress propagates [1, 2, 3, 4]. This force chain network reflects the specific packing of the system, which is unique for each experiment. Although this leads to significant fluctuations in stress profiles from experiment to experiment, the average stress profiles calculated over an ensemble of similar experiments seem well defined [3]. In particular, the ensemble average of the response to a small localized force, the response function [5], is a bell-shaped curve with scaling properties similar to that of an elastic response function [2, 3, 6, 7]. It is natural, therefore, to ask whether the average stress in granular materials behaves according to elasticity theory. In this Communication we address this question within the context of the Force Chain Model 8] (See [9] for a discussion of a model of masses linked with linear and nonlinear springs.). We find significant deviations from elasticity, except for the case of an isotropic packing with nearly isotropic applied forces.

The recently proposed Force Chain Model (FCM) 8] transforms the singular behavior of stress in states of a granular material into a continuum theory by averaging over an ensemble of states. This is done by writing a Master equation for the average density of force chains, allowing force chains to propagate, split and merge [8, 10]. Previously, the response function of the FCM was calculated in three different fashions: simulating force chain propagation in small quenched disordered media [8]; calculating the constitutive relation for granular materials on large scales using a splitting-only variant of the force chain model [8]; and linearizing a specific discretized version of the model around a homogeneous solution [10]. The response functions calculated by the first two methods agreed qualitatively with the experimentally measured response [2, 3], exhibiting a bell shaped peak, while the third method gave a tran- mediate length scale [10].

A priori, there are reasons to expect granular materials to behave non-elastically: They cannot sustain tensile stresses, they rearrange when external loads are changed, and they have no equilibrium stress-free state with respect to which to define a displacement field (See [9] for a different view on these issues.). Despite these considerations, the central result of Reference [8] was that, in the absence of force chain mergers, granular materials behave in a quasi-elastic manner on large length scales. In this Communication, we argue that the effect of force chain mergers is to change this: Generically, granular materials do not behave elastically. The apparent elastic-like behavior found in experiments [2, 3] is restricted to specific packing geometries (i.e. isotropic) and to specific configurations of applied loads (i.e. nearisotropic).

Stress profiles in the FCM may be calculated in three ways, each adapted to a different length scale: Monte-Carlo simulation on small scales; numerical solution of the discretized model of Ref. [10] on intermediate scales, and calculation of the constitutive relation of the full FCM on large scales. We will discuss the latter two methods; the simulation results will be presented in [11]. We will show that observed deviations from elasticity [3] may be understood in the context of the FCM.

In the framework of the FCM, a force chain is characterized by its intensity, $f$ (the pressure exerted on each grain along the force chain), its direction, $\hat{n}$, (which is determined with respect to the applied force on the boundary), and its position, $\vec{r}$. There are four events that involve the creation or annihilation a force chain $\{f, \hat{n}\}$ at $\vec{r}[10$ : it can split; another force chain can split, creating it as one of its offspring; it can merge with another and so be annihilated; or two other force chains can 
for the force chain density, $P \equiv P(f, \hat{n}, \vec{r})[8,10]$ :

$$
\begin{aligned}
& \hat{n} \cdot \vec{\nabla} P=-\frac{1}{\lambda} P \\
& +\frac{2}{\lambda} \int P_{1} \psi_{0} \delta\left(f \hat{n}-\left(f_{1} \hat{n}_{1}+f_{2} \hat{n}_{2}\right)\right) d \underline{f_{1}} \underline{d f_{2}} \\
& -Q P \int P_{1} \varphi_{0} \delta\left(f_{2} \hat{n}_{2}-\left(f_{1} \hat{n}_{1}+f \hat{n}\right)\right) d \underline{f_{1}} d \underline{f_{2}} \\
& +\frac{Q}{2} \int P_{1} P_{2} \varphi_{0} \delta\left(f \hat{n}-\left(f_{1} \hat{n}_{1}+f_{2} \hat{n}_{2}\right)\right) d \underline{d \underline{f_{1}}} d \underline{f_{2}} .
\end{aligned}
$$

Here $P_{i}=P\left(f_{i}, \hat{n_{i}}, \vec{r}\right), Q$ is the force chain width 12], $\lambda$ is the splitting mean free path of a force chain, and the functions $\psi_{0}$ and $\varphi_{0}$ are the weights of a splitting/merging event (which depend, in principle, on the directions $\hat{n}, \hat{n}_{1}$, and $\left.\hat{n}_{2}\right) \cdot d f_{j} \equiv$ $d f_{j} d \hat{n}_{j}$, and the delta functions ensure force balance. Throughout this Communication we will assume that when two force chains meet, they merge, i.e. $\varphi_{0}=1$.

Following [8], we define the force chain intensity density, $F(\hat{n}, \vec{r})=\int P(f, \hat{n}, \vec{r}) f d f$ and its angular moments:

$$
\begin{aligned}
J_{\alpha}(\vec{r}) & =a \int n_{\alpha} F(\hat{n}, \vec{r}) d \hat{n} \\
\sigma_{\alpha \beta}(\vec{r}) & =a D \int n_{\alpha} n_{\beta} F(\hat{n}, \vec{r}) d \hat{n} ;
\end{aligned}
$$

$a$ is the grain size, and D the dimension of the system. $\sigma_{\alpha \beta}$ is the local stress tensor [13] and $\vec{J}(\vec{r})$ can be thought of as the average force chain current.

In order to gain insight on the mesoscopic scale of stress profiles in a granular material, we approximate Eq.(1), following Ref. [10]. We employ the discrete ordinate method proposed by Chandrasekhar [14] for solving the Radiative Transfer Equation. This approximates the integrals in Eq. (1) by sums, by discretizing the directions of force chains as: $P(f, \hat{n}, \vec{r})=\sum_{i=1}^{6} P_{i} \delta\left(f-f^{*}\right) \delta\left(\hat{n}-\hat{n}_{i}\right)$ where $\hat{n}_{i}=\left(\cos \theta_{i}, \sin \theta_{i}\right), i=1, . ., 6$, and $\theta_{i+1}-\theta_{i}=\frac{\pi}{3}$ (with $\theta_{7} \equiv \theta_{1}$ ). The $P_{i}$ 's are six different functions representing the weights of the force chains propagating in directions $\hat{n}_{i}$. Note that the choice $\theta_{i+1}-\theta_{i}=\frac{\pi}{3}$ implies that all forces have the same intensity, $f^{*}$, in order to satisfy force balance. Substituting this into Eq. (1) results in six coupled differential equations for the six force chain densities, $P_{i}$. Rescaling $P_{i} \rightarrow \frac{1}{\lambda Q} P_{i}$ and $\vec{r} \rightarrow \frac{\vec{r}}{\lambda}$ we arrive at the dimensionless equations [10]:

$$
\begin{aligned}
\hat{n}_{i} \cdot \nabla P_{i} & =-P_{i}+P_{i+1}+P_{i-1} \\
& +P_{i+1} P_{i-1}-P_{i+2} P_{i}-P_{i-2} P_{i}
\end{aligned}
$$

Note that these equations are written for isotropic homogeneous assemblies, since the mean free path is assumed constant
In Ref. 10] homogeneous solutions of the form $\left\{P_{j}\right\}=\left\{q, q^{2}, q, q^{-1}, q^{-2}, q^{-1}\right\}$ for any $q$ were considered; however there are others, for example: $\left\{P_{j}\right\}=\left\{q^{-1}, 1, q, q, 1, q^{-1}\right\}$. In [10], Eq. (4) was solved by linearization around the first homogeneous solution, and, remarkably, a double peaked response was shown to emerge at intermediate depths. It is important to understand what physical conditions these solutions correspond to. Let us begin by choosing $q=1$ in either of the homogeneous solutions; this means that the force chain density, and therefore the stress, is uniform throughout. This represents the case of an isotropic array stressed hydrostatically, which is the reference frame for response function experiments. In the case that $q \neq 1$ we have some predetermined relation between force chain densities in different directions at the same position which is unphysical for an isotropic material.

Numerical solutions of the descretized model, Eq. (4), were obtained using a second-order accurate finite-difference approximation and solving the resulting nonlinear algebraic system of equations iteratively 11. In Figure 11 the response to a normal force as calculated by this model is plotted [15]. The peak is symmetric, as expected. In contrast, the response to a tilted force (Figure 2

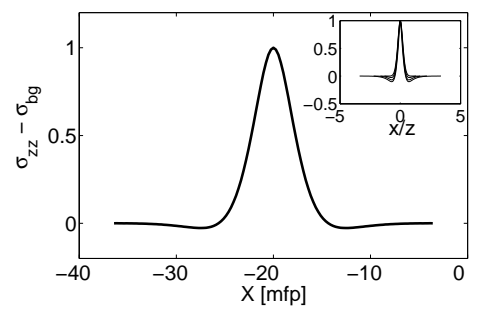

FIG. 1: The response function as calculated with boundary conditions: $P_{i}=0$. The width of the response scales linearly with depth as seen in the insert, where the response function at various depths is plotted; the curves normalized by peak height. See footnote [15].

shows the pressure profile on a plane normal to the applied force.) is asymmetric. The width at half maximum of both functions increases linearly, in accord with the experiments of Ref. 3]. The bell shaped peak of the response to the normal force is in agreement with the elastic-like behavior ascribed to granular materials (see e.g. [8, 16, 17]). However, the asymmetric response to the tilted force deviates from the elasticity prediction [18].

An explanation for the deviation from elasticity of the response function to a tilted force can be found in Eq. (11) which connects the force chain density in one direction with that in any other direction. This means that if all force chains arriving at the surface are canceled (the grains rearrange to 


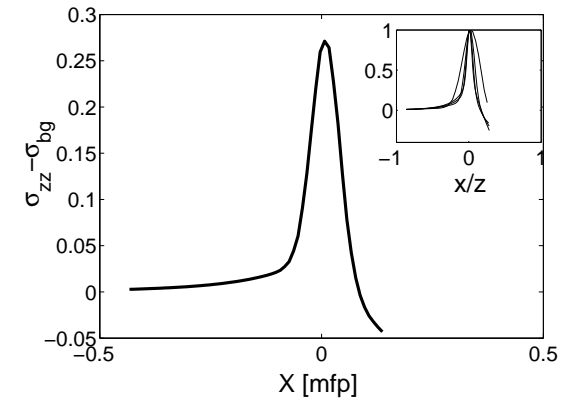

FIG. 2: The response of a granular assembly to a force tilted $60^{\circ}$ with respect to the free surface. Insert: Stress normalized by peak height, showing linear scaling with depth. See footnote [15].

have zero stress at a free surface, for instance) the total force chain density will be zero in the vicinity of that surface. For an elastic material, however, it is possible to have no strain in the direction perpendicular to the surface and a finite strain parallel to the surface, since the strain components are independent. It is noteworthy that this deviation from elasticity is observed also in the splitting-only version of the force chain model (see 19]).

One of the fundamental characteristics of the full force chain model is its non-linear nature (see Equations (1) and (4)). In order to estimate the effect of this non-linearity we tested superposition by comparing the response to two different perturbations, first applied simultaneously and then applied separately. Figure [3(a) presents the response to two forces applied close to one another, and Figure 3 b) two forces applied further apart. It is clear that while the effect of force chain interaction is significant in the former case, it is negligible in the latter.
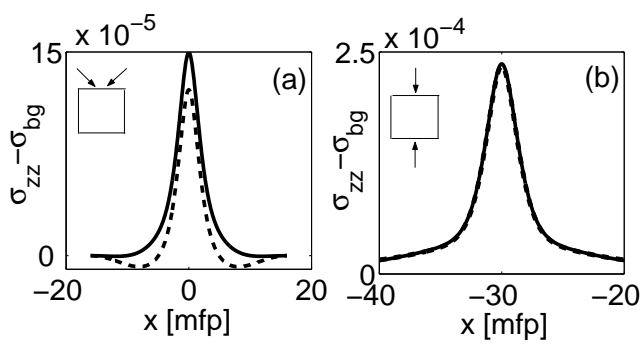

FIG. 3: A comparison between the response to two forces applied simultaneously (dashed line), and applied separately (solid line). a) the forces are applied close to each other, b) the forces are applied at a distance. Lack of superposition obtains for (a) but not for (b). See footnote 15].

While these results are suggestive, the discretized model gives incomplete understanding because all forces in the system are equal. Thus, to better our understanding of the effect of force chain mergers we calculated the constitutive relation on the macroscopic scale by calculating angular moments of Eq. (11), in the spirit of Ref. [8]. Multiplying Eq. (1) by $f \hat{n}$ and integrating over $f \hat{n}$ we arrive at the force balance equation:

$$
\vec{\nabla} \cdot \sigma=\vec{F}_{0}
$$

where $\vec{F}_{0}$ is an external body force. In order to calculate the second moment of Eq. (1), one has to compute the integral:

$I \equiv \int P_{1} P_{2} f \hat{n}_{\alpha} \hat{n}_{\beta} \varphi_{0} \delta\left(f \hat{n}-\left(f_{1} \hat{n}_{1}+f_{2} \hat{n}_{2}\right)\right) d \underline{f} d \underline{f_{1}} d \underline{f_{2}}$

It has been shown both in experiments 20, 21, 22 and in simulations 23 that the probability distribution of forces, $P(f)$, has a maximum, and decays exponentially for larger forces. Thus, we approximate the above integral by considering small deviations of $f_{1}$ and $f_{2}$ from the intensity, $f_{\max }$ at which the $P(f)$ is maximal. That is, we write:

$$
\begin{aligned}
& f_{1}=f_{\text {max }}+\delta f_{1} \\
& f_{2}=f_{\text {max }}+\delta f_{2}
\end{aligned}
$$

and neglect terms with high orders of $\delta f_{i}$ in $I$. Moreover, we assume near-isotropy, by expanding $F(\hat{n}, \vec{r})$ in spherical harmonics and keeping only the terms

$$
a F(\hat{n}, \vec{r}) \simeq p+D \hat{n} \cdot \vec{J}+\frac{D+2}{2} \hat{n} \cdot \tilde{\sigma} \cdot \hat{n}
$$

where $\tilde{\sigma}$ is the traceless part of the stress tensor and $p=\frac{1}{D} \operatorname{Tr}\{\sigma\}$ is the pressure. This gives a constitutive relation [11]:

$$
\begin{aligned}
\sigma_{\alpha \beta} & =A\left[B(\phi) \vec{\nabla} \cdot \vec{J} \delta_{\alpha \beta}+J_{\alpha \beta}\right]-C\langle\hat{n}\rangle \cdot \vec{J}(\vec{r}) \delta_{\alpha \beta} \\
& -D\left(\langle\hat{n}\rangle_{\alpha} J(\vec{r})_{\beta}+\langle\hat{n}\rangle_{\beta} J(\vec{r})_{\alpha}\right)
\end{aligned}
$$

where $\langle n\rangle \quad=\equiv \int \hat{n} P(f, \hat{n}, \vec{r}) d \underline{f}, \quad J_{\alpha \beta} \equiv$ $\frac{1}{2}\left(\partial_{\alpha} J_{\beta}+\partial_{\beta} J_{\alpha}\right)$, and whose constants $A, C, D$ are determined by the specifics of the granular packing. The function $B(\phi)$ depends as well on the force chain density $\phi(\vec{r}) \equiv \int P(f, \hat{n}, \vec{r}) d f[11]$.

For nearly homogeneous and isotropic systems, the terms which are products of $\vec{J}$ and $\langle\hat{n}\rangle$ are smaller than terms linear in $\vec{J}$. If they may be ignored, the constitutive relation reduces to:

$$
\sigma_{\alpha \beta}=A\left[B(\phi) \vec{\nabla} \cdot \vec{J} \delta_{\alpha \beta}+J_{\alpha \beta}\right]
$$

This equation is formally equivalent to the constitutive relation of conventional elasticity [18]. Therefore, we can define two pseudo-elastic moduli: The pseudo-Poisson ratio, $\nu=\frac{B}{1+2 B}$, and the pseudo-Young modulus, $E=A(1+\nu)$. These depend not only on the geometry of the pile, but also 
Therefore, the pseudo elastic behavior obtains only for nearly homogenous systems.

Generally speaking, the constitutive relations calculated by the force chain model (Eq. (7)), are different from those of conventional elasticity; in particular, they are nonlinear. This nonlinearity is somewhat subtle, and holds for the ensemble averaged stresses. For a given packing, which does not change upon application of external forces, it is clear that superposition must hold, since the grainscale equations of force balance are linear. Consider, however, the ensemble of stress states which are consistent with given set of external forces $\left\{\mathcal{F}_{1}\right\}$. We believe that this ensemble is statistically different from that ensemble which is compatible with a different set of external forces $\left\{\mathcal{F}_{2}\right\}$, even if $\left\{\mathcal{F}_{1}\right\}$ and $\left\{\mathcal{F}_{2}\right\}$ are very similar. Physically, this is reflected in the fragility [24] of the material: rearrangements occur when the external conditions are changed. Thus, the FCM predicts that if an ensemble averaged stress field is measured, then granular materials will exhibit nonlinearity in its response.

In this Communication we have dealt mainly with the ensemble average of the stress profile in granular materials. However, the singularity of the force chains and the wide distribution of force chain intensity measured 1] suggest that it might be interesting to study force fluctuations, and the effect of friction, in the framework of the FCM. As in References [8, 10], the existence of force chains, in the sense of a reasonably straight line of grains in contact, was assumed. It remains to be seen under what conditions this assumption is reasonable. We expect that for very hard grains (more precisely, small stresses compared to the grain compressibility), force chains will exist. If this is the case, the effect of friction would be to change the details of the packing obtained, such as the scattering mean free path and persistence length, but not undermine the existence of force chains (Indeed, grains are frictional in all real experiments [2, 3, 4]. Furthermore, friction might stabilize a force chain network, by allowing it to bear loads which would otherwise be "incompatible", this in turn can lead to a larger load regime for which the granular material responds linearly.

DL gratefully acknowledges support from the US-Israel Binational Science Foundation (grant 9900235) and the Israel Science Foundation (grant $88 / 02$ ). It is a pleasure to acknowledge useful and interesting discussions with G. Bunin, P. Claudin, C. Goldenfeld, I. Goldhirsch, S. Rahav, J. Socolar, and Y. Srebro.
[1] C.H. Liu et al., Science 269, 513 (1995).

[2] J. Geng et al., Phys. Rev Lett. 87, 035506 (2001).

[3] J. Geng et al., Physica D 182, 274 (2003).

[4] P. Dantu, Geotechnique 18, 50 (1968).

[5] In $2 \mathrm{D}$, the stress $\sigma\left(x, z=z_{0}\right)$ at depth $z_{0}$ as a function of the transverse coordinate $x$.

[6] G. Reydellet and E. Clément, Phys. Rev. Lett. 86, 3308 (2001).

[7] S.N. Coppersmith, C.-h. Liu, S Majumdar, O. Narayan, and T.A. Witten, Phys Rev E 53, 4673 (1996)

[8] J.-P. Bouchaud, P. Claudin, D. Levine, and M. Otto, Eur. Phys. J. E 4, 451 (2001).

[9] C.Goldenberg and I. Goldhirsch, Eur. Phys. J. E 9, 245-251 (2002). C.Goldenberg and I. Goldhirsch, Phys. Rev. Lett. 89, 084302 (2002).

[10] J.E.S Socolar, D.G. Schaeffer, and P. Claudin, Eur. Phys. J. E 7, 353 (2002).

[11] Y. Roichman, I. Yavneh, and D. Levine (in preparation).

[12] $Q$, which has dimensions of length $h^{d-1}$ in $d$ dimensions, is analogous to the chain cross section.

[13] S.F. Edwards, Physica A 249, 226 (1998).

[14] S. Chandrasekhar, Radiative Transfer, 3 ed. (Pergamon press, Oxford, 1986).

[15] Note that there a background threshold stress $\sigma_{b g}$ to the stress distributions of the discretized model, which corresponds to the fixed point solution $P_{j}=$ 1. This is subtracted in the figures.

[16] S.B. Savage, in 3rd Int. Conf. of Powders and Grains, edited by R.P. Behringer and J.T. Jenkins (Balkema, Rotterdam, Durham NC, 1997).

[17] P.G. de Gennes, Rev. Mod. Phys. 71, s374 (1999).

[18] L.D. Landau and E.M. Lifshitz, Theory of elasticity, 3 ed. (Pergamon press, Oxford, 1986).

[19] Y. Roichman, G. Bunin, and D. Levine (in preparation).

[20] D.M. Mueth, H.M. Jaeger, and S.R. Nagel, Phys. Rev. E 57, 3164 (1998).

[21] R. Brockbank, J.M Huntly, and R.C. Ball, J. Phys. II, France 7, 1521 (1997).

[22] D.L. Blair, N.W. Mueggenburg, A.H. Marshall, H.M. Jaeger, and S.R. Nagel, Phys. Rev. E 63, 041304 (2001).

[23] F. Radjai, M. Jean, J.-J. Moreau, and S. Roux, Phys. Rev. Lett. 77, 274 (1996). F. Radjai, D.E. Wolf, M. Jean, and J.-J. Moreau, Phys. Rev. Lett. 80, 61 (1998).

[24] M.E. Cates, J.P. Wittamer, J.P. Bouchaud, and P. Claudin, Physica A 263, 354 (1999). 